\title{
STRONG MORITA EQUIVALENCE OF HIGHER-DIMENSIONAL NONCOMMUTATIVE TORI. II
}

\author{
GEORGE A. ELLIOTT AND HANFENG LI
}

\begin{abstract}
We show that two $C^{*}$-algebraic noncommutative tori are strongly Morita equivalent if and only if they have isomorphic ordered $K_{0}$-groups and centers, extending N. C. Phillips's result in the case that the algebras are simple. This is also generalized to the twisted group $C^{*}$-algebras of arbitrary finitely generated abelian groups.
\end{abstract}

\section{INTRODUCTION}

Let $n \geq 2$ and denote by $\mathcal{T}_{n}$ the space of $n \times n$ real skew-symmetric matrices. For each $\theta \in \mathcal{T}_{n}$, the corresponding $n$-dimensional $\left(C^{*}\right.$ algebraic) noncommutative torus $A_{\theta}$ is defined as the universal $C^{*}$ algebra generated by unitaries $U_{1}, \cdots, U_{n}$ satisfying the relations

$$
U_{k} U_{j}=e\left(\theta_{k j}\right) U_{j} U_{k},
$$

where $e(t)=e^{2 \pi i t}$. Noncommutative tori are one of the canonical examples in noncommutative differential geometry $[32,9]$.

One may also consider the smooth version $A_{\theta}^{\infty}$ of a noncommutative torus, which is the algebra of formal series

$$
\sum c_{j_{1}, \cdots, j_{n}} U_{1}^{j_{1}} \cdots U_{n}^{j_{n}}
$$

where the coefficient function $\mathbb{Z}^{n} \ni\left(j_{1}, \cdots, j_{n}\right) \mapsto c_{j_{1}, \cdots, j_{n}}$ belongs to the Schwartz space $\mathcal{S}\left(\mathbb{Z}^{n}\right)$. This is the space of smooth elements of $A_{\theta}$ for the canonical action of $\mathbb{T}^{n}$ on $A_{\theta}$.

A notion of Morita equivalence of $C^{*}$-algebras (as an analogue of Morita equivalence of unital rings [1, Chapter 6]) was introduced by Rieffel in $[27,30]$. This is now often called strong Morita equivalence or Rieffel-Morita equivalence. Strongly Morita equivalent $C^{*}$-algebras

Date: February 15, 2008.

2000 Mathematics Subject Classification. Primary 46L87; Secondary 58B34.

This research was supported by a grant from the Natural Sciences and Engineering Research Council of Canada, held by the first named author. 
share a lot in common such as equivalent categories of Hilbert $C^{*}$ modules, isomorphic $K$-groups, etc., and hence are usually thought of as having the same geometry.

In [35] Schwarz introduced the notion of complete Morita equivalence of smooth noncommutative tori, which includes strong Morita equivalence of the corresponding $C^{*}$-algebras, but is stronger (as it also involves the smooth structure). This has important application in $\mathrm{M}$ (atrix) theory [35, 19].

A natural question is to classify noncommutative tori and their smooth counterparts up to the various notions of Morita equivalence. Such results are of some interest from the point of view of physics [10,35]. In the case $n=2$ this was done by Rieffel [29]. In this case it does not matter what kind of Morita equivalence one considers: there is a (densely defined) action of the group $\mathrm{GL}(2, \mathbb{Z})$ on $\mathcal{T}_{2}$, and two matrices in $\mathcal{T}_{2}$ give rise to Morita equivalent noncommutative tori or smooth noncommutative tori if and only if they are in the same orbit of this action, and also if and only if the ordered $K_{0}$-groups of the algebras are isomorphic. The higher dimensional case is much more complicated and there are examples showing that the smooth counterparts of two strongly Morita equivalent noncommutative tori may fail to be Morita equivalent (as unital $\mathbb{C}$-algebras) [14] (see also [33, 6]).

After the work of Rieffel, Schwarz, and the second-named author in $[33,35,21]$ (see also [40]) it is now known that $n$-dimensional smooth noncommutative tori are classified up to complete Morita equivalence by the (densely defined) $\mathrm{SO}(n, n \mid \mathbb{Z})$ action on $\mathcal{T}_{n}$ introduced in [33], as a generalization of the $\mathrm{GL}(2, \mathbb{Z})$ action in the 2 -dimensional case. In $[14]$ we showed that, in the generic case, $n$-dimensional smooth noncommutative tori are classified up to Morita equivalence (as unital $\mathbb{C}$-algebras) in the same way.

Phillips showed that two simple noncommutative tori of dimension $n$ are strongly Morita equivalent if and only if the exponential maps on $\Lambda^{\text {even }}\left(\mathbb{Z}^{n}\right)$ associated to the skew-symmetric matrices have the same range up to a scalar multiplication [25, Theorem 3.11], or equivalently, their ordered $K_{0}$-groups are isomorphic (the equivalence of these two formulations follows from [13, Theorem 3.1] and Proposition 4.1 below). In this paper we shall complete the classification of noncommutative tori with respect to strong Morita equivalence. It turns out that in the general (nonsimple) case one needs to know, in addition to the ordered $K_{0}$-group of the algebra, only the center. (Since the center is isomorphic to $C\left(\mathbb{T}^{k}\right)$ for some nonnegative integer $k$, it is enough to know the dimension of $\mathbb{T}^{k}$. See Theorem 1.1 and Remark 4.4.) 
We shall also do more than classify the noncommutative tori (as defined above), up to strong Morita equivalence. Note that $n$-dimensional noncommutative tori are exactly the twisted group $C^{*}$-algebras of $\mathbb{Z}^{n}$ (see Subsection 2.2). We shall obtain the classification, up to strong Morita equivalence, of the twisted group $C^{*}$-algebras of arbitrary finitely generated abelian groups. These are exactly the $C^{*}$-algebras admitting ergodic actions of compact abelian Lie groups [23].

Theorem 1.1. Two twisted group $C^{*}$-algebras of finitely generated abelian groups, in particular two noncommutative tori, are strongly Morita equivalent if and only if they have isomorphic ordered $K_{0}$-groups and centers.

It is known that two unital $C^{*}$-algebras are strongly Morita equivalent if and only if they are Morita equivalent as unital $\mathbb{C}$-algebras $[4$, Theorem 1.8]. Thus Theorem 1.1 also classifies the twisted group $C^{*}$ algebras of finitely generated abelian groups up to Morita equivalence as unital $\mathbb{C}$-algebras.

The opposite algebra $A^{\text {op }}$ of a $C^{*}$-algebra $A$ is the algebra $A$ with the multiplication reversed but all other operations, including the scalar multiplication, the same. (It is still a $C^{*}$-algebra.) A unital $C^{*}$-algebra may not be strongly Morita equivalent to its opposite algebra (see [24] for some interesting examples), and a smooth noncommutative torus may not be Morita equivalent to its opposite algebra (see the discussion after Theorem 1.1 in [14]). It is a long-standing open question whether every noncommutative torus is isomorphic to its opposite algebra. This is now known to be the case for simple noncommutative tori [25, Corollary 3.14]. Since the property of admitting an ergodic action of a fixed compact group is preserved under passing to the opposite algebra, the class of the twisted group $C^{*}$-algebras of finitely generated abelian groups is closed under the operation of taking the opposite algebra. As a consequence of Theorem 1.1, we obtain the answer to the easier version of the question above with strong Morita equivalence in place of isomorphism:

Corollary 1.2. Any twisted group $C^{*}$-algebra of a finitely generated abelian group is strongly Morita equivalent to its opposite algebra.

There are two main ingredients in our proof of Theorem 1.1. The first is that, geometrically speaking, every smooth noncommutative torus is completely Morita equivalent to the Cartesian product of a smooth simple noncommutative torus and an ordinary smooth torus. This result depends on Rieffel's construction of finitely generated projective modules over noncommutative tori in $[31]$ and also on the $\operatorname{SO}(n, n \mid \mathbb{Z})$ 
action on $\mathcal{T}_{n}$ introduced by Rieffel and Schwarz in [33]. The second ingredient is Phillips's classification, up to strong Morita equivalence, of simple noncommutative tori, which as mentioned above depends on his structure theorem for these algebras [25, Theorem 3.8]. To extend Theorem 1.1 to the twisted group $C^{*}$-algebras of arbitrary (countable) discrete abelian groups, one might need to extend these results of Phillips, Rieffel, and Schwarz to the infinite dimensional case.

This paper is organized as follows. We review the basic definitions and facts concerning complete Morita equivalence and the twisted group algebras in Section 2. We prove the above-mentioned complete Morita equivalence result in Section 3 using the main result of [21]. Theorem 1.1 is proved in Section 4 in the case of noncommutative tori. In Section 5 we extend the methods and results of Sections 3 and 4 to the twisted group algebras of arbitrary finitely generated abelian groups, obtaining in particular Theorem 1.1 in full generality.

This work was carried out while $\mathrm{H}$. Li was at the University of Toronto.

\section{Preliminaries}

2.1. Complete Morita equivalence. In this subsection we recall Schwarz's definition of complete Morita equivalence and note that it passes to quotients.

We refer the reader to [1, Sections 21 and 22] for details on algebraic Morita equivalence, to [27, 28, 30] for strong Morita equivalence, and to [35] and [19, Section 7.2] for complete Morita equivalence.

Let $A$ and $B$ be pre- $C^{*}$-algebras, i.e., dense sub-*-algebras of $C^{*}$ algebras. A strong Morita equivalence $A$ - $B$-bimodule is an $A$ - $B$-bimodule ${ }_{A} E_{B}$ with an $A$-valued inner product ${ }_{A}\langle\cdot, \cdot\rangle$ and a $B$-valued inner product $\langle\cdot, \cdot\rangle_{B}$ satisfying certain conditions (see [27, Definition 6.10] for detail; there an equivalence bimodule is called an imprimitivity bimodule). $E$ has a norm defined by $\|x\|:=\left\|_{A}\langle x, x\rangle\right\|^{1 / 2}=\left\|\langle x, x\rangle_{B}\right\|^{1 / 2}$ for $x \in E[28$, Proposition 3.1]. The completion of $E$ is a strong Morita equivalence bimodule between the completions of $A$ and $B$. By [28, Theorem 3.1, Lemma 3.1] (note that the condition there that $A$ and $B$ be $C^{*}$-algebras is unnecessary), there is a bijective correspondence between the lattice of closed two-sided ideals $J$ of $B$ and the lattice of closed $A$-B-submodules $Y$ of $E$ via $Y=\overline{E J}=\{y \in E$ : $\langle x, y\rangle_{B} \in J$ for all $\left.x \in E\right\}$ and $J=\overline{\langle E, Y\rangle}$. By symmetry a similar correspondence holds for the lattice of closed two-sided ideals of $A$. Moveover, if $K(J)$ is the ideal of $A$ corresponding to $Y$ (the submodule corresponding to $J$ as above), then the $A$-valued inner product on $E$ 
drops to an $A / K(J)$-valued inner product on $E / Y$, and the $B$-valued inner product on $E$ drops to a $B / J$-valued product on $E / Y$, so that $E / Y$ becomes a strong Morita equivalence $A / K(Y)-B / J$-bimodule [28, Corollary 3.2]. $A$ and $B$ are strongly Morita equivalent if there exists a strong Morita equivalence $A$ - $B$-bimodule. In particular, if $A$ and $B$ are strongly Morita equivalent, then so also are their completions.

Throughout the rest of this section we shall assume further that $A$ and $B$ are unital and spectrally invariant, i.e., an element of $A$ (resp. $B$ ) is invertible in $A$ (resp. $B$ ) if it is invertible in the completion of $A$ (resp. $B$ ). Let ${ }_{A} E_{B}$ be a strong Morita equivalence $A$ - $B$-bimodule. Then ${ }_{A}\langle E, E\rangle=A$ and $\langle E, E\rangle_{B}=B$. Furthermore, ${ }_{A} E_{B}$ is an algebraic Morita equivalence $A$ - $B$-bimodule (see the proof of $[4$, Theorem $1.8])$; that is, $A=\operatorname{End}\left(E_{B}\right), B=\operatorname{End}\left({ }_{A} E\right)$, and ${ }_{A} E$ and $E_{B}$ are finitely generated projective modules and are generators in the sense that ${ }_{A} A$ and $B_{B}$ are direct summands of direct sums of finitely many copies of ${ }_{A} E$ and $E_{B}$ respectively. It is also easily checked that in the correspondence between closed two-sided ideals $J$ of $B$ and closed $A$ $B$-submodules $Y$ of $E$ described above, one has $Y=E J$ (using the fact that $E_{B}$ is a finitely generated projective $B$-module) and $J=\langle E, Y\rangle_{B}$ (using $Y=E J$ and $\langle E, E\rangle_{B}=B$ ).

Suppose that a Lie algebra $L_{B}$ (resp. $L_{A}$ ) acts on $B$ (resp. $A$ ) as *-derivations. A (Hermitian) connection on $E_{B}[8]$ is a linear map $\nabla: L_{B} \rightarrow \operatorname{Hom}_{\mathbb{C}}(E)$ satisfying the Leibniz rule:

$$
\begin{aligned}
\nabla_{X}(y b) & =\left(\nabla_{X} y\right) b+y\left(\delta_{X} b\right), \\
\delta_{X}\langle x, y\rangle_{B} & =\left\langle\nabla_{X} x, y\right\rangle_{B}+\left\langle x, \nabla_{X} y\right\rangle_{B},
\end{aligned}
$$

for all $X \in L_{B}, b \in B$ and $x, y \in E$, where $\delta_{X}$ is the derivation of $B$ corresponding to $X$. A connection $\nabla$ is said to have constant curvature if $\left[\nabla_{X}, \nabla_{X^{\prime}}\right]-\nabla_{\left[X, X^{\prime}\right]}$ is a scalar multiplication for all $X, X^{\prime} \in L_{B}$. E is said to be a complete Morita equivalence bimodule [35] (this is called a gauge Morita equivalence bimodule in $[38,19,37])$ between $\left(A, L_{A}\right)$ and $\left(B, L_{B}\right)$ if there are constant curvature connections for ${ }_{A} E$ and $E_{B}$ respectively and a Lie algebra isomorphism from $L_{B}$ onto $L_{A}$ such that the diagram

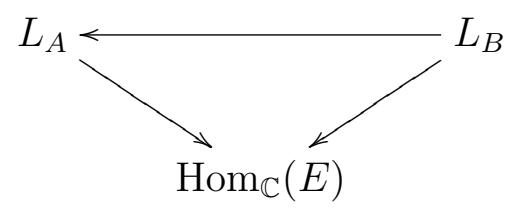

commutes. Let $J, Y$, and $K(J)$ be as above. If a Lie subalgebra $L_{B / J}$ of $L_{B}$ leaves $J$ invariant, then one checks easily that $L_{B / J}$ and $L_{A / K(J)}$ also leave $Y$ and $K(J)$ respectively invariant, where $L_{A / K(J)}$ denotes 
the image of $L_{B / J}$ in $L_{A}$, and that the actions of $L_{B}$ and $L_{A}$ on $B$ and $A$ drop to actions of $L_{B / J}$ and $L_{A / K(J)}$ on $B / J$ and $A / K(J)$ respectively such that $E / Y$ is a complete Morita equivalence bimodule between $\left(A / K(J), L_{A / K(L)}\right)$ and $\left(B / J, L_{B / J}\right) .\left(A, L_{A}\right)$ and $\left(B, L_{B}\right)$ are said to be completely Morita equivalent if there exists a complete Morita equivalence bimodule between them (see [36, 37] for a more general notion called Morita equivalence of $Q$-algebras).

2.2. Twisted group algebras. In this subsection we shall recall basic definitions and facts about the twisted group $C^{*}$-algebras [42] and smooth twisted group algebras of finitely generated abelian groups.

Let $G$ be a finitely generated abelian group, and let $\sigma$ be a 2cocycle on $G$ (with values in the unit circle group $\mathbb{T}$ ), i.e., a map $G \times G \rightarrow \mathbb{T}$ such that $\sigma\left(g_{1}, g_{2}\right) \sigma\left(g_{1} g_{2}, g_{3}\right)=\sigma\left(g_{1}, g_{2} g_{3}\right) \sigma\left(g_{2}, g_{3}\right)$ for all $g_{1}, g_{2}, g_{3} \in G$. The twisted group $C^{*}$-algebra $C^{*}(G ; \sigma)$ is the universal $C^{*}$-algebra generated by unitaries $u_{g}$ for $g \in G$ subject to the condition $u_{g} \cdot u_{h}=\sigma(g, h) u_{g h}$. Since $G$ is finitely generated and abelian, the dual group $\hat{G}$ is a Lie group. Because of the universal property of $C^{*}(G ; \sigma), \hat{G}$ has a canonical strongly continuous action $\alpha$ on $C^{*}(G ; \sigma)$ determined by $\alpha_{x}\left(u_{g}\right)=x(g) u_{g}$. This action is ergodic in the sense that the fixed point elements are exactly the scalar multiples of the unit. The smooth twisted group algebra $S(G ; \sigma)$ is the algebra of smooth elements of $C^{*}(G ; \sigma)$ with respect to this action. It is a spectrally invariant dense sub-*-algebra of $C^{*}(G ; \sigma)$. The Lie algebra $\operatorname{Lie}(\hat{G})$ acts on $S(G ; \sigma)$ as $*$-derivations. Throughout the rest of this paper, we shall use this Lie algebra action on $S(G ; \sigma)$, and when we talk about complete Morita equivalence between two $(S(G ; \sigma)$, Lie $(\hat{G}))$ 's we shall simply say complete Morita equivalence between the $S(G ; \sigma)$ 's.

Two 2-cocycles $\sigma$ and $\sigma^{\prime}$ on $G$ are said to be cohomologous if there is a 1-cochain $\lambda$ on $G$, i.e., a map $\lambda$ from $G$ to $\mathbb{T}$, such that $\sigma^{\prime}(g, h)=$ $\lambda_{g} \lambda_{h} \lambda_{g h}^{-1} \sigma(g, h)$ for all $g, h \in G$. In this case, there is a natural $\hat{G}$ equivariant $*$-isomorphism from $C^{*}(G ; \sigma)$ onto $C^{*}\left(G ; \sigma^{\prime}\right)$. A map $\sigma$ from $G \times G$ to $\mathbb{T}$ is said to be a bicharacter if $\sigma(g, \cdot)$ and $\sigma(\cdot, g)$ are homomorphisms from $G$ to $\mathbb{T}$ for each $g \in G$. Clearly, every bicharacter is a 2-cocycle. Conversely, every 2-cocycle is cohomologous to a bicharacter [18, Theorem 7.1].

A bicharacter $\sigma^{\prime}$ on $G$ is said to be skew-symmetric if $\sigma(g, g)=1$ for all $g \in G$, which implies that $\sigma(g, h) \sigma(h, g)=1$ for all $g, h \in G$. Associated to any 2-cocycle $\sigma$ on $G$, there is a skew-symmetric bicharacter $\sigma^{*}$ on $G$ defined by $\sigma^{*}(g, h)=\sigma(g, h) \sigma(h, g)^{-1}$ for all $g, h \in G$. Two 2-cocycles $\sigma$ and $\sigma^{\prime}$ on $G$ are cohomologous exactly if $\sigma^{*}=\left(\sigma^{\prime}\right)^{*}$ 
[23, Proposition 3.2]. Associated to a 2-cocycle $\sigma$ on $G$, there is also a homomorphism $\beta_{\sigma}$ from $G$ to $\hat{G}$ defined by $\beta_{\sigma}(g)(\cdot)=\sigma^{*}(g, \cdot)$ for all $g \in G$. Denote by $H_{\sigma}$ the kernel of $\beta_{\sigma}$. The center of $C^{*}(G ; \sigma)$ (resp. $S(G ; \sigma)$ ) is the closed linear span of $u_{g}, g \in H_{\sigma}$, in $C^{*}(G ; \sigma)$ (resp. $S(G ; \sigma)$ ) and is isomorphic to the algebra of ( $\mathbb{C}$-valued) continuous (resp. smooth) functions on $\widehat{H_{\sigma}}$ via the Fourier transform. $\sigma$ is said to be nondegenerate if $H_{\sigma}=\{0\}$. It is known that $\sigma$ is nondegenerate exactly if $C^{*}(G ; \sigma)$ is simple [39, Theorem 3.7], and also exactly if $S(G ; \sigma)$ is simple (see [5, Lemma 3.2] and Theorem 13 of [17] and the remark following).

When $G=\mathbb{Z}^{n}$ for $n \geq 0$, any $\theta \in \mathcal{T}_{n}$ gives rise to a skew-symmetric bicharacter $\sigma_{\theta}$ on $\mathbb{Z}^{n}$ defined by $\sigma_{\theta}(g, h)=e^{\pi i g \theta h^{t}}$. One has $A_{\theta}=$ $C^{*}\left(\mathbb{Z}^{n} ; \sigma_{\theta}\right)$ and $A_{\theta}^{\infty}=S\left(\mathbb{Z}^{n} ; \sigma_{\theta}\right)$ through the identification of $U_{j}$ with $u_{e_{j}}$ for $1 \leq j \leq n$, where $U_{1}, \cdots, U_{n}$ are the canonical generators of $A_{\theta}$ and $e_{1}, \cdots, e_{n}$ are the canonical basis elements of $\mathbb{Z}^{n}$. On the other hand, any bicharacter $\sigma$ on $\mathbb{Z}^{n}$ may be written as $\sigma(g, h)=e^{2 \pi i g \Theta h^{t}}$ for some $n \times n$ real matrix $\Theta$. Set $\theta=\Theta-\Theta^{t}$. Then $\theta$ is in $\mathcal{T}_{n}$. Note that $\sigma$ and $\sigma_{\theta}$ are cohomologous via the 1-cochain $\lambda$ given by $\lambda_{g}=e^{\pi i g \Theta g^{t}}$. Therefore, every 2-cocycle on $\mathbb{Z}^{n}$ is cohomologous to $\sigma_{\theta}$ for some $\theta \in \mathcal{T}_{n}$. Thus, noncommutative tori and smooth noncommutative tori are exactly all the twisted group $C^{*}$-algebras and smooth twisted group algebras, respectively, of torsion-free finitely generated abelian groups.

\section{Complete Morita equivalence}

As well as the case $n \geq 2$, we shall consider also 1-dimensional and 0-dimensional noncommutative tori below, though they are actually commutative. An element $\theta$ in $\mathcal{T}_{n}$ for $n>0$ is said to be nondegenerate if any element $X$ in $\mathbb{Z}^{n}$ with $X \theta \in \mathbb{Z}^{n}$ is 0 . As a convention, let us say that $\theta$ is nondegenerate if $n=0$. Then $\theta$ is nondegenerate exactly if the 2-cocycle $\sigma_{\theta}$ defined in Subsection 2.2 is nondegenerate, and also exactly if $A_{\theta}$ is simple, and also exactly if $A_{\theta}^{\infty}$ is simple. The main result of this section is the following

Theorem 3.1. For any $\theta \in \mathcal{T}_{n}$, the smooth noncommutative torus $A_{\theta}^{\infty}$ is completely Morita equivalent to $A_{\theta^{\prime}}^{\infty}$ for some $\theta^{\prime} \in \mathcal{T}_{n}$ such that

$$
\theta^{\prime}=\left(\begin{array}{ll}
0 & 0 \\
0 & \tilde{\theta}
\end{array}\right)
$$

where $\tilde{\theta}$ belongs to $\mathcal{T}_{k}$ for some $0 \leq k \leq n$ and is nondegenerate. 
Remark 3.2. Note that $A_{\theta}^{\infty}$ has a natural Fréchet topology (see for instance [14, Example 3.1]). It turns out that $A_{\theta^{\prime}}^{\infty}$ is the topological tensor product [41, Chapter 43] of the Fréchet algebras $A_{\tilde{\theta}}^{\infty}$ and $C^{\infty}\left(\mathbb{T}^{n-k}\right)$, where $C^{\infty}\left(\mathbb{T}^{n-k}\right)$ is the algebra of smooth functions on $\mathbb{T}^{n-k}$. Thus, in geometric language, Theorem 3.1 says that every smooth noncommutative torus is completely Morita equivalent to the Cartesian product of a smooth simple noncommutative torus and an ordinary smooth torus.

Denote by $\mathrm{O}(n, n \mid \mathbb{R})$ the group of linear transformations of the space $\mathbb{R}^{2 n}$ preserving the quadratic form $x_{1} x_{n+1}+x_{2} x_{n+2}+\cdots+x_{n} x_{2 n}$, and by $\mathrm{SO}(n, n \mid \mathbb{Z})$ the subgroup of $\mathrm{O}(n, n \mid \mathbb{R})$ consisting of matrices with integer entries and determinant 1.

Following [33] let us write the elements of $\mathrm{O}(n, n \mid \mathbb{R})$ in $2 \times 2$ block form:

$$
g=\left(\begin{array}{ll}
A & B \\
C & D
\end{array}\right)
$$

Then $A, B, C$, and $D$ are arbitrary $n \times n$ matrices satisfying

$$
A^{t} C+C^{t} A=0=B^{t} D+D^{t} B, \quad A^{t} D+C^{t} B=I .
$$

The group $\mathrm{SO}(n, n \mid \mathbb{Z})$ has a partial action on $\mathcal{T}_{n}$ [33], defined by

$$
g \theta=(A \theta+B)(C \theta+D)^{-1}
$$

whenever $C \theta+D$ is invertible. For each $g \in \mathrm{SO}(n, n \mid \mathbb{Z})$ this action is defined on a dense open subset of $\mathcal{T}_{n}$ (see the discussion before [33, Theorem]). (The set of maximal homeomorphisms between dense open subsets of a Hausdorff space can be made into a group, with the natural composition law consisting of taking ordinary composition, to the extent defined (always on a dense open subset), and then extending to the largest open subset on which this map is a homeomorphism onto another open subset (the largest such open subset always exists). The map from $\mathrm{SO}(n, n \mid \mathbb{Z})$ to the group of what might be called (maximal essential) partial homeomorphisms of $\mathcal{T}_{n}$ is a group homomorphism.)

Theorem 3.1 follows immediately from Proposition 3.3 below and [21, Theorem 1.1].

Proposition 3.3. Let $\theta \in \mathcal{T}_{n}$. Then there exists $g \in \mathrm{SO}(n, n \mid \mathbb{Z})$ such that $g \theta$ is defined and

$$
g \theta=\left(\begin{array}{cc}
0 & 0 \\
0 & \tilde{\theta}
\end{array}\right)
$$

where $\tilde{\theta}$ belongs to $\mathcal{T}_{k}$ for some $0 \leq k \leq n$ and is nondegenerate. 
Example 3.4. Let $\gamma$ be a real number and $m$ be a nonzero integer. Consider the matrix

$$
\theta=\left(\begin{array}{ccc}
0 & -3 / m & -2 / m \\
3 / m & 0 & \gamma \\
2 / m & -\gamma & 0
\end{array}\right) \in \mathcal{T}_{3}
$$

Consider $g=\left(\begin{array}{ll}A & B \\ C & D\end{array}\right) \in \mathrm{SO}(3,3 \mid \mathbb{Z})$ where

$$
\begin{array}{ccc}
A=\left(\begin{array}{ccc}
m & 0 & 0 \\
0 & -2 & 3 \\
0 & -m & m
\end{array}\right), & B=\left(\begin{array}{lll}
0 & 3 & 2 \\
0 & 0 & 0 \\
1 & 0 & 0
\end{array}\right) \\
C=\left(\begin{array}{ccc}
0 & 1 & -1 \\
0 & 0 & 0 \\
1 & 0 & 0
\end{array}\right), & D=\left(\begin{array}{lll}
0 & 0 & 0 \\
0 & 1 & 1 \\
0 & 0 & 0
\end{array}\right) .
\end{array}
$$

Then $g \theta$ is defined, and

$$
g \theta=\left(\begin{array}{ccc}
0 & 0 & 0 \\
0 & 0 & m \gamma \\
0 & -m \gamma & 0
\end{array}\right)
$$

Let us establish some preliminary results to prepare for the proof of Proposition 3.3. Associated to the quadratic form $x_{1} x_{n+1}+x_{2} x_{n+2}+$ $\cdots+x_{n} x_{2 n}$ there is a symmetric bilinear form $\langle\cdot, \cdot\rangle$ on $\mathbb{R}^{2 n}$ given by

$$
\left\langle\left(x_{1}, \cdots, x_{2 n}\right),\left(y_{1}, \cdots, y_{2 n}\right)\right\rangle=\sum_{j=1}^{n}\left(x_{j} y_{n+j}+x_{n+j} y_{j}\right) .
$$

The elements of $\mathrm{O}(n, n \mid \mathbb{R})$ are exactly those linear transformations of $\mathbb{R}^{2 n}$ preserving $\langle\cdot, \cdot\rangle$. Let us say that a basis $e_{1}, \cdots, e_{n}, f_{1}, \cdots, f_{n}$ for $\mathbb{Z}^{2 n}$ is compatible with the form $\langle\cdot, \cdot\rangle$ if

$$
\left\langle e_{i}, e_{j}\right\rangle=\left\langle f_{i}, f_{j}\right\rangle=0 \quad \text { and } \quad\left\langle e_{i}, f_{j}\right\rangle=\delta_{i, j}
$$

for all $1 \leq i, j \leq n$. The standard basis of $\mathbb{Z}^{2 n}$ is a compatible one.

Lemma 3.5. Let $M$ be a direct summand of $\mathbb{Z}^{2 n}$ such that $M$ is isotropic, i.e., $\langle M, M\rangle=0$. Then any basis for $M$ extends to a basis for $\mathbb{Z}^{2 n}$ compatible with $\langle\cdot, \cdot\rangle$.

Proof. Note that the pairing between $M$ and $0^{n} \oplus \mathbb{Z}^{n}$ under $\langle\cdot, \cdot\rangle$ induces a homomorphism $0^{n} \oplus \mathbb{Z}^{n} \rightarrow \operatorname{Hom}(M, \mathbb{Z})$. Denote by $W$ the kernel of this homomorphism. Then $M+W$ is also isotropic.

Denote by $\pi$ the projection of $\mathbb{Z}^{2 n}$ onto $\mathbb{Z}^{n} \oplus 0^{n}$. Similarly, the pairing between $\pi(M)$ and $0^{n} \oplus \mathbb{Z}^{n}$ under $\langle\cdot, \cdot\rangle$ induces a surjective 
homomorphism $0^{n} \oplus \mathbb{Z}^{n} \rightarrow \operatorname{Hom}(\pi(M), \mathbb{Z})$. Note that the kernel of this homomorphism is also $W$. Thus, $n=\operatorname{rank}(\pi(M))+\operatorname{rank}(W)$.

Since $\pi(M)$ is a free abelian group, we can find a homomorphism $\psi: \pi(M) \rightarrow M$ such that $\pi \circ \psi$ is the identity map on $\pi(M)$. Then the restriction of $\pi$ to $\psi(\pi(M))$ is injective, and hence $\psi(\pi(M)) \cap\left(0^{n} \oplus\right.$ $\left.\mathbb{Z}^{n}\right)=\psi(\pi(M)) \cap \operatorname{ker} \pi=\{0\}$. In particular, $\psi(\pi(M)) \cap W=\{0\}$. Consequently,

$$
\begin{aligned}
\operatorname{rank}(M+W) & \geq \operatorname{rank}(\psi(\pi(M)))+\operatorname{rank}(W) \\
& =\operatorname{rank}(\pi(M))+\operatorname{rank}(W)=n .
\end{aligned}
$$

Consider the set $\tilde{M}$ of elements of $\mathbb{Z}^{2 n}$ some multiple of which by a nonzero integer is in $M+W$. Then $\tilde{M}$ is an isotropic subgroup of $\mathbb{Z}^{2 n}$ with rank at least $n$. By the elementary divisor theorem [20, Theorem III.7.8], $\tilde{M}$ is a direct summand of $\mathbb{Z}^{2 n}$, and any basis for $M$ extends to a basis for $\tilde{M}$. Replacing $M$ by $\tilde{M}$, we may assume that $\operatorname{rank}(M) \geq n$.

The pairing between $M$ and $\mathbb{Z}^{2 n}$ under $\langle\cdot, \cdot\rangle$ induces a homomorphism $\varphi: \mathbb{Z}^{2 n} \rightarrow \operatorname{Hom}(M, \mathbb{Z})$. Note that for any $x \in \mathbb{Z}^{2 n}$, if $x / m$ is not in $\mathbb{Z}^{2 n}$ for every integer $m \geq 2$, then there exists $y \in \mathbb{Z}^{2 n}$ with $\langle x, y\rangle=1$. Using again the elementary divisor theorem, one sees easily that $\varphi$ is surjective. Since $M$ is contained in the kernel of $\varphi$, we obtain

$$
\begin{aligned}
2 n & =\operatorname{rank}(\operatorname{ker}(\varphi))+\operatorname{rank}(\operatorname{Hom}(M, \mathbb{Z})) \\
& \geq \operatorname{rank}(M)+\operatorname{rank}(\operatorname{Hom}(M, \mathbb{Z}))=2 \cdot \operatorname{rank}(M) .
\end{aligned}
$$

Therefore, $\operatorname{rank}(\operatorname{ker}(\varphi))=\operatorname{rank}(M)=n$. Using the elementary divisor theorem one more time, one sees that $M$ is a direct summand of $\operatorname{ker}(\varphi)$. It follows that the kernel of $\varphi$ is exactly $M$.

Let $e_{1}, \cdots, e_{n}$ be a basis for $M$. Choose $h_{1}, \cdots, h_{n}$ in $\mathbb{Z}^{2 n}$ such that $\varphi\left(h_{1}\right), \cdots, \varphi\left(h_{n}\right)$ is the dual basis of $e_{1}, \cdots, e_{n}$. Then the subgroup $P$ of $\mathbb{Z}^{2 n}$ generated by $h_{1}, \cdots, h_{n}$ maps isomorphically onto $\operatorname{Hom}(M, \mathbb{Z})$ under $\varphi$, and hence $\mathbb{Z}^{2 n}=M \oplus P$. In other words, $e_{1}, \cdots, e_{n}, h_{1}, \cdots, h_{n}$ is a basis for $\mathbb{Z}^{2 n}$. Note that for any $x \in \mathbb{Z}^{2 n},\langle x, x\rangle$ is an even integer. Define $f_{j} \in \mathbb{Z}^{2 n}$ inductively by

$$
f_{j}=h_{j}-\frac{1}{2}\left\langle h_{j}, h_{j}\right\rangle e_{j}-\sum_{k=1}^{j-1}\left\langle h_{j}, f_{k}\right\rangle e_{k} .
$$

Then, clearly, $e_{1}, \cdots, e_{n}, f_{1}, \cdots, f_{n}$ is a basis for $\mathbb{Z}^{2 n}$ compatible with $\langle\cdot, \cdot\rangle$.

Remark 3.6. Let $R$ be a principal entire ring [20, page 86] with characteristic not equal to 2 . Then Lemma 3.5 holds with $\mathbb{Z}$ replaced by $R$. 
Lemma 3.7. For any $n \times n$ matrix $A$ with entries in $\mathbb{C}$, there exists a function $\zeta:\{1, \cdots, n\} \rightarrow\{1,-1\}$ such that

$$
\operatorname{det}(A-\operatorname{diag}(\zeta(1), \cdots, \zeta(n))) \neq 0 .
$$

Proof. We prove the assertion by induction on $n$. The case $n=1$ is trivial. Suppose that the assertion holds for $n=k$ and $A$ is a $(k+1) \times(k+1)$ matrix. Denote by $B$ the $k \times k$ upper left corner of $A$. Then we can find a function $\zeta:\{1, \cdots, k\} \rightarrow\{1,-1\}$ such that $\operatorname{det}(B-$ $\operatorname{diag}(\zeta(1), \cdots, \zeta(k))) \neq 0$. Define functions $\zeta^{+}, \zeta^{-}:\{1, \cdots, k+1\} \rightarrow$ $\{1,-1\}$ extending $\zeta$ with $\zeta^{ \pm}(k+1)= \pm 1$. Observing that the matrices $A-\operatorname{diag}\left(\zeta^{+}(1), \cdots, \zeta^{+}(k+1)\right)$ and $A-\operatorname{diag}\left(\zeta^{-}(1), \cdots, \zeta^{-}(k+1)\right)$ differ at only one entry, we have

$$
\begin{aligned}
& \operatorname{det}\left(A-\operatorname{diag}\left(\zeta^{-}(1), \cdots, \zeta^{-}(k+1)\right)\right)- \\
& \operatorname{det}\left(A-\operatorname{diag}\left(\zeta^{+}(1), \cdots, \zeta^{+}(k+1)\right)\right) \\
= & 2 \cdot \operatorname{det}(B-\operatorname{diag}(\zeta(1), \cdots, \zeta(k))) \neq 0 .
\end{aligned}
$$

Thus at least one of $\zeta^{+}$and $\zeta^{-}$satisfies the requirement. This finishes the induction step.

Lemma 3.8. Let $e_{1}, \cdots, e_{n}, f_{1}, \cdots, f_{n}$ be a basis for $\mathbb{Z}^{2 n}$ compatible with $\langle\cdot, \cdot\rangle$. Let $V$ be an $n$-dimensional isotropic linear subspace of $\mathbb{R}^{2 n}$, i.e., $\langle V, V\rangle=0$. Then we can choose $\eta_{j}$ from $e_{j}$ and $f_{j}$ for each $1 \leq j \leq n$ in such a way that $\operatorname{span}_{\mathbb{R}}\left(\eta_{1}, \cdots, \eta_{n}\right) \cap V=\{0\}$.

Proof. Set $u_{j}=e_{j}+f_{j}$ and $v_{j}=e_{j}-f_{j}$ for each $j$. Set $W_{1}=$ $\operatorname{span}_{\mathbb{R}}\left(u_{1}, \cdots, u_{n}\right)$ and $W_{2}=\operatorname{span}_{\mathbb{R}}\left(v_{1}, \cdots, v_{n}\right)$. With respect to the basis $u_{1}, \cdots, u_{n}, v_{1}, \cdots, v_{n}$, the bilinear form $\langle\cdot, \cdot\rangle$ on $\mathbb{R}^{2 n}$ gives rise to the quadratic form

$$
\mathbb{R}^{2 n} \ni \sum x_{j} u_{j}+\sum y_{j} v_{j} \mapsto \sum x_{j}^{2}-\sum y_{j}^{2} .
$$

Thus, the restriction of $\langle\cdot, \cdot\rangle$ to $W_{2}$ is negative definite. Since $V$ is isotropic, $V \cap W_{2}=\{0\}$. Therefore, $V \subseteq \mathbb{R}^{2 n}=W_{1} \oplus W_{2}$ is the graph of a linear map $\varphi: W_{1} \rightarrow W_{2}$. Denote by $A$ the matrix of $\varphi$ with respect to the bases $u_{1}, \cdots, u_{n}$ and $v_{1}, \cdots, v_{n}$. Choose $\zeta$ as in Lemma 3.7 applied to $A$. Then $\varphi\left(u_{1}\right)-\zeta(1) v_{1}, \cdots, \varphi\left(u_{n}\right)-\zeta(n) v_{n}$ are linearly independent. Note that $u_{1}+\varphi\left(u_{1}\right), \cdots, u_{n}+\varphi\left(u_{n}\right)$ is a basis for $V$. It follows easily that $\operatorname{span}_{\mathbb{R}}\left(u_{1}+\zeta(1) v_{1}, \cdots, u_{n}+\zeta(n) v_{n}\right) \cap V=\{0\}$. Now we may just take $\eta_{j}$ to be $\frac{1}{2}\left(u_{j}+\zeta(j) v_{j}\right)$ for each $j$.

The next lemma is a consequence of [40, Corollary 2.3]. For the convenience of the reader, we give a direct proof here. 
Lemma 3.9. Let $g=\left(\begin{array}{ll}A & B \\ C & D\end{array}\right) \in \mathrm{O}(n, n \mid \mathbb{Z})$. If $C \theta+D$ is invertible for some $\theta \in \mathcal{T}_{n}$, so that $g \theta$ is defined in the sense of (1), then $g$ is in $\mathrm{SO}(n, n \mid \mathbb{Z})$.

Proof. Suppose that $C \theta+D$ is invertible and $g$ is not in $\operatorname{SO}(n, n \mid \mathbb{Z})$. Let $\varphi$ denote the linear transformation of $\mathbb{R}^{2 n}$ exchanging the 1 st and the $(n+1)$-st coordinates. Then $\varphi$ preserves the quadratic form $x_{1} x_{n+1}+$ $x_{2} x_{n+2}+\cdots+x_{n} x_{2 n}$ and has determinant -1 . Thus the matrix $h$ corresponding to $\varphi$ (with respect to the standard basis of $\mathbb{R}^{2 n}$ ) is in $\mathrm{O}(n, n \mid \mathbb{Z})$ but not in $\mathrm{SO}(n, n \mid \mathbb{Z})$. Therefore, $h g$ is in $\mathrm{SO}(n, n \mid \mathbb{Z})$, and in particular $h g$ acts on a dense open subset of $\mathcal{T}_{n}$. Perturbing $\theta$ slightly, we may assume then that $(h g)(\theta)$ is defined and that $g(\theta)$ is still defined in the sense of (1). Set $\theta^{\prime}=g(\theta)$. Then (by matrix algebra) $\theta=g^{-1}\left(\theta^{\prime}\right)$ and hence (in the same way) $h g\left(g^{-1}\left(\theta^{\prime}\right)\right)$ is defined in the sense of (1). It follows (in the same way) that $h\left(\theta^{\prime}\right)$ is defined in the sense of (1). But it is easy to see that $h$ does not act on any element of $\mathcal{T}_{n}$ in the sense of (1). Thus we get a contradiction. Therefore, $g$ is in $\mathrm{SO}(n, n \mid \mathbb{Z})$.

We are ready to prove Proposition 3.3.

Proof of Proposition 3.3. Denote by $\alpha_{1}, \cdots, \alpha_{n}, \beta_{1}, \cdots, \beta_{n}$ the standard basis of $\mathbb{R}^{2 n}$. Denote by $\varphi$ the linear map $0^{n} \oplus \mathbb{R}^{n} \rightarrow \mathbb{R}^{n} \oplus 0^{n}$ whose matrix with respect to the bases $\beta_{1}, \cdots, \beta_{n}$ and $\alpha_{1}, \cdots, \alpha_{n}$ is $\theta$. Since $\theta$ is skew-symmetric, the graph $V$ of $\varphi$ is an $n$-dimensional isotropic linear subspace of $\mathbb{R}^{2 n}$. Denote by $M$ the intersection of $V$ and $\mathbb{Z}^{2 n}$. Using the elementary divisor theorem [20, Theorem III.7.8], one sees easily that $M$ is a direct summand of $\mathbb{Z}^{2 n}$. By Lemma 3.5 we can find a basis $e_{1}, \cdots, e_{n}, f_{1}, \cdots, f_{n}$ for $\mathbb{Z}^{2 n}$ compatible with $\langle\cdot, \cdot\rangle$ such that $f_{1}, \cdots, f_{n-k}$ is a basis for $M$. Observing that $f_{1}, \cdots, f_{n-k} \in V$, by Lemma 3.8 we may assume that $\operatorname{span}_{\mathbb{R}}\left(e_{1}, \cdots, e_{n}\right) \cap V=\{0\}$. Then $V$ is the graph of a linear map $\psi: \operatorname{span}_{\mathbb{R}}\left(f_{1}, \cdots, f_{n}\right) \rightarrow \operatorname{span}_{\mathbb{R}}\left(e_{1}, \cdots, e_{n}\right)$. Denote by $\theta^{\prime}$ the matrix of $\psi$ with respect to the bases $f_{1}, \cdots, f_{n}$ and $e_{1}, \cdots, e_{n}$. Since $f_{1}, \cdots, f_{n-k} \in V$, we have $\psi\left(f_{1}\right)=\cdots=\psi\left(f_{n-k}\right)=0$, and hence the first $n-k$ columns of $\theta^{\prime}$ are 0 . Since $V$ is isotropic and $e_{1}, \cdots, e_{n}, f_{1}, \cdots, f_{n}$ is a basis for $\mathbb{Z}^{2 n}$ compatible with $\langle\cdot, \cdot\rangle$, one sees easily that $\theta^{\prime}$ is skew-symmetric. Therefore, $\theta^{\prime}=\left(\begin{array}{cc}0 & 0 \\ 0 & \tilde{\theta}\end{array}\right)$ for some $\tilde{\theta} \in \mathcal{T}_{k}$. Set $g$ to be the $2 n \times 2 n$ matrix such that

$$
\left(e_{1}, \cdots, e_{n}, f_{1}, \cdots, f_{n}\right) g=\left(\alpha_{1}, \cdots, \alpha_{n}, \beta_{1}, \cdots, \beta_{n}\right) .
$$

Since $g$ takes a compatible basis to another one, it is in $\mathrm{O}(n, n \mid \mathbb{Z})$. Using the expressions of elements of $V$ in terms of $\theta$ and $\theta^{\prime}$ respectively, 
a simple calculation shows that $g \theta$ is defined in the sense of (1) and $g \theta=\theta^{\prime}$. By Lemma 3.9, $g$ is in $\mathrm{SO}(n, n \mid \mathbb{Z})$.

It remains to show that $\tilde{\theta}$ is nondegenerate. Let $\left(y_{n-k+1}, \cdots, y_{n}\right) \in \mathbb{Z}^{k}$ be such that $\tilde{\theta}\left(y_{n-k+1}, \cdots, y_{n}\right)^{t}$ has integral entries. Then

$$
\sum_{j=n-k+1}^{n} y_{j} f_{j}+\psi\left(\sum_{j=n-k+1}^{n} y_{j} f_{j}\right) \in V \cap \mathbb{Z}^{2 n}=M .
$$

Since $f_{1}, \cdots, f_{n-k}$ is a basis for $M$, we get $y_{n-k+1}=\cdots=y_{n}=0$. Therefore, $\tilde{\theta}$ is nondegenerate.

\section{Morita equivalence}

In this section we prove Theorem 1.1 in the case of noncommutative tori.

We discuss first how to see whether two noncommutative tori have isomorphic ordered $K_{0}$-groups. (See [2, Section 6] for basics on ordered $K_{0}$-groups.) For any $\theta \in \mathcal{T}_{n}, A_{\theta}$ has a canonical tracial state $\tau_{\theta}$ given by the integration over the canonical action of $\widehat{\mathbb{Z}^{n}}$. By [13, Lemma 2.3], all tracial states on $A_{\theta}$ induce the same homomorphism from $K_{0}\left(A_{\theta}\right)$ to $\mathbb{R}$, which we denote by $\omega_{\theta}$. By [13, Theorem 3.1], $\omega_{\theta}\left(K_{0}\left(A_{\theta}\right)\right)$ is the subgroup of $\mathbb{R}$ generated by 1 and the numbers $\sum_{\xi}(-1)^{|\xi|} \prod_{s=1}^{m} \theta_{j_{\xi(2 s-1)} j_{\xi(2 s)}}$ for $1 \leq j_{1}<j_{2}<\cdots<j_{2 m} \leq n$, where the sum is taken over all elements $\xi$ of the permutation group $S_{2 m}$ such that $\xi(2 s-1)<\xi(2 s)$ for all $1 \leq s \leq m$ and $\xi(1)<\xi(3)<\cdots<\xi(2 m-1)$. $\theta$ is said to be rational if its entries are all rational numbers; otherwise it is said to be nonrational. Clearly $\theta$ is rational if and only if $\omega_{\theta}\left(K_{0}\left(A_{\theta}\right)\right)$ has rank 1 .

Proposition 4.1. Let $\theta_{j} \in \mathcal{T}_{n_{j}}$ for $j=1,2$. Then $A_{\theta_{1}}$ and $A_{\theta_{2}}$ have isomorphic ordered $K_{0}$-groups if and only if $\omega_{\theta_{2}}\left(K_{0}\left(A_{\theta_{2}}\right)\right)=\mu \omega_{\theta_{1}}\left(K_{0}\left(A_{\theta_{1}}\right)\right)$ for some real number $\mu>0$ and either $n_{1}=n_{2}$ or $n_{1}+n_{2}=1$.

Proof. From the Pimsner-Voiculescu exact sequence [26] one knows that $K_{0}\left(A_{\theta_{j}}\right)$ is a free abelian group of rank 1 or $2^{n_{j}-1}$ depending on whether $n_{j}=0$ or $n_{j}>0$.

We prove first the "only if" part. Comparing the ranks of the $K_{0^{-}}$ groups we see that either $n_{1}=n_{2}$ or $n_{1}+n_{2}=1$ (i.e., one of $n_{1}$ and $n_{2}$ is 0 and the other is 1$)$. Every unital $C^{*}$-algebra admitting an ergodic action of a compact abelian group is nuclear [23, Lemma 6.2], [12, Proposition 3.1]. Since $A_{\theta_{j}}$ admits an ergodic action of $\mathbb{T}^{n_{j}}$, it is nuclear and hence is exact. For a unital $C^{*}$-algebra $A$, a state on the scaled ordered $K_{0}$-group $\left(K_{0}(A)_{+}, K_{0}(A),\left[1_{A}\right]\right)$ is a positive unital homomorphism from $\left(K_{0}(A)_{+}, K_{0}(A),\left[1_{A}\right]\right)$ to $\left(\mathbb{R}_{+}, \mathbb{R}, 1\right)$. When $A$ is exact, every state on $\left(K_{0}(A)_{+}, K_{0}(A),\left[1_{A}\right]\right)$ comes from 
a tracial state on $A[3$, Corollary 3.4], [16, Theorem 9.2]. Therefore, $\left(K_{0}\left(A_{\theta_{j}}\right)_{+}, K_{0}\left(A_{\theta_{j}}\right),\left[1_{A_{\theta_{j}}}\right]\right)$ has a unique state, which is exactly $\omega_{\theta_{j}}$. Take an order isomorphism $\psi$ from $\left(K_{0}\left(A_{\theta_{1}}\right)_{+}, K_{0}\left(A_{\theta_{1}}\right)\right)$ onto $\left(K_{0}\left(A_{\theta_{2}}\right)_{+}, K_{0}\left(A_{\theta_{2}}\right)\right)$. Then $\omega_{\theta_{2}} \circ \psi$ is a nontrivial positive homomorphism from $\left(K_{0}\left(A_{\theta_{1}}\right)_{+}, K_{0}\left(A_{\theta_{1}}\right)\right)$ to $\left(\mathbb{R}_{+}, \mathbb{R}\right)$. Set $\mu$ equal to the value of $\left[1_{A_{\theta_{1}}}\right]$ under $\omega_{\theta_{2}} \circ \psi$. Then $\mu>0$ and $\frac{1}{\mu}\left(\omega_{\theta_{2}} \circ \psi\right)$ is a state on $\left(K_{0}\left(A_{\theta_{1}}\right)_{+}, K_{0}\left(A_{\theta_{1}}\right),\left[1_{A_{\theta_{1}}}\right]\right)$. Consequently, $\frac{1}{\mu}\left(\omega_{\theta_{2}} \circ \psi\right)=\omega_{\theta_{1}}$. Evaluating both sides on $K_{0}\left(A_{\theta_{1}}\right)$ we get $\omega_{\theta_{2}}\left(K_{0}\left(A_{\theta_{2}}\right)\right)=\mu \omega_{\theta_{1}}\left(K_{0}\left(A_{\theta_{1}}\right)\right)$.

Next we prove the "if" part. Note that $\omega_{\theta_{j}}\left(K_{0}\left(A_{\theta_{j}}\right)\right)$ is a torsion-free finitely generated abelian group, and hence is a free abelian group. Taking a lifting of $\omega_{\theta_{j}}\left(K_{0}\left(A_{\theta_{j}}\right)\right)$ in $K_{0}\left(A_{\theta_{j}}\right)$ and identifying this lifting with $\omega_{\theta_{j}}\left(K_{0}\left(A_{\theta_{j}}\right)\right)$, we may assume that $K_{0}\left(A_{\theta_{j}}\right)=\operatorname{ker}\left(\omega_{\theta_{j}}\right) \oplus \omega_{\theta_{j}}\left(K_{0}\left(A_{\theta_{j}}\right)\right)$ and that $\omega_{\theta_{j}}$ is exactly the projection onto the second summand. Now we need to distinguish the cases $\theta_{j}$ is rational or nonrational. Suppose that both $\theta_{1}$ and $\theta_{2}$ are nonrational. Then $n_{1}=n_{2}$. By [31, Theorem 6.1], $\left(K_{0}\left(A_{\theta_{j}}\right)\right)_{+}$consists of exactly the elements of $K_{0}\left(A_{\theta_{j}}\right)$ on which $\omega_{\theta_{j}}$ is strictly positive, together with 0 . The multiplication by $\mu$ is an order isomorphism from $\omega_{\theta_{1}}\left(K_{0}\left(A_{\theta_{1}}\right)\right)$ onto $\omega_{\theta_{2}}\left(K_{0}\left(A_{\theta_{2}}\right)\right)$. Then $\operatorname{ker}\left(\omega_{\theta_{1}}\right)$ and $\operatorname{ker}\left(\omega_{\theta_{2}}\right)$ have the same rank. Taking any isomorphism from $\operatorname{ker}\left(\omega_{\theta_{1}}\right)$ onto $\operatorname{ker}\left(\omega_{\theta_{2}}\right)$, we get an order isomorphism from $\left(K_{0}\left(A_{\theta_{1}}\right)_{+}, K_{0}\left(A_{\theta_{1}}\right)\right)$ onto $\left(K_{0}\left(A_{\theta_{2}}\right)_{+}, K_{0}\left(A_{\theta_{2}}\right)\right)$, as desired.

Now assume that at least one of $\theta_{1}$ and $\theta_{2}$ is rational. Comparing the ranks of $\omega_{\theta_{1}}\left(K_{0}\left(A_{\theta_{1}}\right)\right)$ and $\omega_{\theta_{2}}\left(K_{0}\left(A_{\theta_{2}}\right)\right)$ we see that both $\theta_{1}$ and $\theta_{2}$ are rational. Note that the partial action of $\mathrm{SO}(n, n \mid \mathbb{Z})$ on $\mathcal{T}_{n}$ preserves rationality. Thus, if we take $\theta=\theta_{j}$ and $n=n_{j}$ in Proposition 3.3, then $\tilde{\theta}$ given in Proposition 3.3 must be rational and hence $k$ given there must be 0 . By [21, Theorem 1.1], $A_{\theta_{j}}^{\infty}$ is completely Morita equivalent to $A_{0_{n \times n}}^{\infty}$, where $0_{n \times n}$ is the zero $n \times n$ matrix. Consequently, $A_{\theta_{1}}$ and $A_{\theta_{2}}$ are Morita equivalent and hence have isomorphic ordered $K_{0^{-}}$ groups. This finishes the proof of Proposition 4.1.

The "only if" part of Proposition 4.1 and the proof of the "if" part show

Corollary 4.2. Let $\theta \in \mathcal{T}_{n}$. Then the following statements are equivalent:

(1) $\theta$ is rational;

(2) $A_{\theta}^{\infty}$ is completely Morita equivalent to $A_{0_{n \times n}}^{\infty}$, where $0_{n \times n}$ is the zero $n \times n$ matrix;

(3) $A_{\theta}$ is Morita equivalent to $A_{0_{n \times n}}=C\left(\mathbb{T}^{n}\right)$, where $C\left(\mathbb{T}^{n}\right)$ is the algebra of continuous functions on $\mathbb{T}^{n}$;

(4) $\omega_{\theta}\left(K_{0}\left(A_{\theta}\right)\right)$ has rank 1 . 
Lemma 4.3. Let $\theta_{1}$ and $\theta_{2}$ in $\mathcal{T}_{n}$ be such that

$$
\theta_{1}=\left(\begin{array}{cc}
0 & 0 \\
0 & \tilde{\theta}_{1}
\end{array}\right) \quad \text { and } \quad \theta_{2}=\left(\begin{array}{cc}
0 & 0 \\
0 & \tilde{\theta}_{2}
\end{array}\right) \text {, }
$$

with $\tilde{\theta}_{1}, \tilde{\theta}_{2} \in \mathcal{T}_{k}$. If the ordered $K_{0}$-groups of $A_{\theta_{1}}$ and $A_{\theta_{2}}$ are isomorphic, then so also are those of $A_{\tilde{\theta}_{1}}$ and $A_{\tilde{\theta}_{2}}$.

Proof. Note that $A_{\theta_{j}}=A_{\tilde{\theta}_{j}} \otimes C\left(\mathbb{T}^{n-k}\right)$. Taking the evaluation at any point of $\mathbb{T}^{n-k}$ we get a unital $*$-homomorphism $\varphi_{j}$ from $A_{\theta_{j}}$ to $A_{\tilde{\theta}_{j}}$. Denote by $\left(\varphi_{j}\right)_{*}$ the induced homomorphism from $K_{0}\left(A_{\theta_{j}}\right)$ to $K_{0}\left(A_{\tilde{\theta}_{j}}\right)$. Then $\omega_{\tilde{\theta}_{j}} \circ\left(\varphi_{j}\right)_{*}$ is exactly $\omega_{\theta_{j}}$. Using the embedding $A_{\tilde{\theta}_{j}} \hookrightarrow A_{\tilde{\theta}_{j}} \otimes$ $C\left(\mathbb{T}^{n-k}\right)=A_{\theta_{j}}$ one sees that $\left(\varphi_{j}\right)_{*}$ is surjective. Thus,

$$
\omega_{\theta_{j}}\left(K_{0}\left(A_{\theta_{j}}\right)\right)=\left(\omega_{\tilde{\theta}_{j}} \circ\left(\varphi_{j}\right)_{*}\right)\left(K_{0}\left(A_{\theta_{j}}\right)\right)=\omega_{\tilde{\theta}_{j}}\left(K_{0}\left(A_{\tilde{\theta}_{j}}\right)\right) .
$$

Now Lemma 4.3 follows from Proposition 4.1.

As we mentioned in Subsection 2.2, the center of $A_{\theta}$ is isomorphic to the algebra of continuous functions on $\widehat{H_{\sigma_{\theta}}}$, and hence depends only on the rank of $H_{\sigma_{\theta}}$, which can be calculated from $\theta$ arithmetically.

We are ready to prove Theorem 1.1 in the case of noncommutative tori.

Proof of Theorem 1.1 in the case of noncommutative tori. The "only if" part follows from the fact that Morita equivalence between unital algebras (or rings) preserves both the ordered $K_{0}$-group and the center [ 1 , Proposition 21.10]. Consider the "if" part. Suppose that $A_{\theta_{1}}$ and $A_{\theta_{2}}$ have isomorphic ordered $K_{0}$-groups and centers. By the "only if" part and Theorem 3.1, we may assume that

$$
\theta_{j}=\left(\begin{array}{cc}
0 & 0 \\
0 & \tilde{\theta}_{j}
\end{array}\right)
$$

for some nondegenerate $\tilde{\theta}_{j} \in \mathcal{T}_{k_{j}}$. Say that $\theta_{j}$ is in $\mathcal{T}_{n_{j}}$. Then $A_{\theta_{j}} \cong$ $C\left(\mathbb{T}^{n_{j}-k_{j}}\right) \otimes A_{\tilde{\theta}_{j}}$. From the Pimsner-Voiculescu exact sequence [26] one knows (as recalled above) that $K_{0}\left(A_{\theta_{j}}\right)$ is a free abelian group of rank 1 or $2^{n_{j}-1}$, depending on whether $n_{j}=0$ or $n_{j}>0$. It follows that either $n_{1}=n_{2}$, or $n_{1}+n_{2}=1$. Note that $A_{\theta}=\mathbb{C}$ if $n_{j}=0$ and $A_{\theta}=C(\mathbb{T})$ if $n_{j}=1$. Therefore we must have $n_{1}=n_{2}$. Also note that the center of $A_{\theta_{j}}$ is isomorphic to $C\left(\mathbb{T}^{n_{j}-k_{j}}\right)$. Thus $k_{1}=k_{2}$. By Lemma $4.3, A_{\tilde{\theta}_{1}}$ and $A_{\tilde{\theta}_{2}}$ have isomorphic ordered $K_{0}$-groups. Since $\tilde{\theta}_{1}$ and $\tilde{\theta}_{2}$ are nondegenerate, both $A_{\tilde{\theta}_{1}}$ and $A_{\tilde{\theta}_{2}}$ are simple. Phillips has shown that simple noncommutative tori are classified up to strong Morita equivalence by their ordered $K_{0}$-groups [25]. Therefore, $A_{\tilde{\theta}_{1}}$ 
and $A_{\tilde{\theta}_{2}}$ are strongly Morita equivalent. Consequently, $A_{\theta_{1}}$ and $A_{\theta_{2}}$ are strongly Morita equivalent. This finishes the proof of Theorem 1.1 in the case of noncommutative tori.

Remark 4.4. In the case of simple noncommutative tori, note that the result of Phillips in [25] refers only to the ordered $K_{0}$-group, not the center. Theorem 1.1 in fact also does this as the center is the scalars in this case. If we consider only noncommutative tori of dimension 2 or 3, then Theorem 1.1 holds without mentioning the centers, since in these cases the dimension of the center is determined by the ordered $K_{0}$-group. The reason in the case of 2 -dimensional noncommutative tori is that in this case the dimension of the center of $A_{\theta}$ is either 2 or 0 , depending as $\omega_{\theta}\left(K_{0}\left(A_{\theta}\right)\right)$ has rank 1 or 2 , as is easily seen from the arithmetical description of $\omega_{\theta}\left(K_{0}\left(A_{\theta}\right)\right)$ given above. The reason in the case of 3 -dimensional noncommutative tori is that in this case the center of $A_{\theta}$ has dimension 3,1 , or 0 , depending as $\omega_{\theta}\left(K_{0}\left(A_{\theta}\right)\right)$ has rank 1, 2, or at least 3. However, Example 4.5 below shows that if we consider $n$-dimensional noncommutative tori for a fixed $n \geq 4$, then Theorem 1.1 does not hold any longer without keeping track of the centers.

Example 4.5. Let $\gamma$ be a real algebraic integer of degree 2 (for example, $\sqrt{2})$. Set

$$
\theta_{1}=\left(\begin{array}{cccc}
0 & \gamma & 0 & 0 \\
-\gamma & 0 & 0 & 0 \\
0 & 0 & 0 & 0 \\
0 & 0 & 0 & 0
\end{array}\right) \quad \text { and } \quad \theta_{2}=\left(\begin{array}{cccc}
0 & \gamma & 0 & 0 \\
-\gamma & 0 & 0 & 0 \\
0 & 0 & 0 & \gamma \\
0 & 0 & -\gamma & 0
\end{array}\right)
$$

Then by the arithmetical description of the range of the trace on $K_{0}$ given above, $\omega_{\theta_{1}}\left(K_{0}\left(A_{\theta_{1}}\right)\right)=\omega_{\theta_{2}}\left(K_{0}\left(A_{\theta_{2}}\right)\right)$, and hence $A_{\theta_{1}}$ and $A_{\theta_{2}}$ have isomorphic ordered $K_{0}$-groups by Proposition 4.1. But the center of $A_{\theta_{1}}$ has dimension 2, while that of $A_{\theta_{2}}$ has dimension 0 .

\section{TWISTED GROUP ALGEBRAS OF FINITELY GENERATED ABELIAN GROUPS}

In this section we extend the results of Sections 3 and 4 to the twisted group algebras of arbitrary finitely generated abelian groups.

Denote by $G_{\text {tor }}$ the torsion subgroup of a finitely generated abelian group $G$. The rank of $G$ is the dimension of the $\mathbb{Q}$-vector space $G \otimes_{\mathbb{Z}}$ $\mathbb{Q}$. Recall that a 2-cocycle on $G$ is nondegenerate if the subgroup $H_{\sigma}$ defined in Subsection 2 is $\{0\}$. The following result is a generalization of Theorem 3.1. 
Theorem 5.1. Let $\sigma$ be a 2-cocycle on a finitely generated abelian group $G$. Then there exist a finitely generated abelian group $G^{\prime}=$ $G_{1}^{\prime} \oplus G_{2}^{\prime}$ and a skew-symmetric bicharacter $\sigma^{\prime}$ on $G^{\prime}$ such that $\operatorname{rank}(G)=$ $\operatorname{rank}\left(G^{\prime}\right), G_{2}^{\prime}$ is torsion-free, $\sigma^{\prime}\left(G_{1}^{\prime}, G^{\prime}\right)=1$, the restriction of $\sigma^{\prime}$ to $G_{2}^{\prime}$ is nondegenerate, and $S\left(G^{\prime} ; \sigma^{\prime}\right)$ is completely Morita equivalent to $S(G ; \sigma)$.

Proof. Since $G$ is finitely generated and abelian, we can find a nonnegative integer $n$ and a surjective homomorphism $\psi$ from $\mathbb{Z}^{n}$ to $G$. Then the pull-back $\psi^{*}(\sigma)$ of $\sigma$ under $\psi$ is a 2-cocycle on $\mathbb{Z}^{n}$. In Subsection 2.2 we noticed that there exists an element $\theta$ of $\mathcal{T}_{n}$ such that $\psi^{*}(\sigma)$ is cohomologous to $\sigma_{\theta}$ via a 1-cochain $\lambda$ on $\mathbb{Z}^{n}$. By Theorem 3.1, $A_{\theta}^{\infty}$ is completely Morita equivalent to $A_{\theta^{\prime}}^{\infty}$ for some $\theta^{\prime} \in \mathcal{T}_{n}$ of the form

$$
\theta^{\prime}=\left(\begin{array}{ll}
0 & 0 \\
0 & \tilde{\theta}
\end{array}\right)
$$

where $\tilde{\theta} \in \mathcal{T}_{k}$ for some $0 \leq k \leq n$ and is nondegenerate. Let $A_{\theta^{\prime}}^{\infty} E_{A_{\theta}^{\infty}}$ be a complete Morita equivalence $A_{\theta^{\prime}}^{\infty}-A_{\theta}^{\infty}$-bimodule with constantcurvature connections on $E_{A_{\theta}^{\infty}}$ and $A_{\theta^{\prime}}^{\infty} E$ and a Lie algebra isomorphism $\phi: \operatorname{Lie}\left(\widehat{\mathbb{Z}^{n}}\right) \rightarrow \operatorname{Lie}\left(\widehat{\mathbb{Z}^{n}}\right)$ as in Subsection 2.1.

In Subsection 2.1 we mentioned that complete Morita equivalence passes to quotient algebras (with the actions of certain Lie subalgebras). We shall find an ideal $J$ of $A_{\theta}^{\infty}$ such that $A_{\theta}^{\infty} / J \cong S(G ; \sigma)$ and identify the corresponding ideal $K(J)$ of $A_{\theta^{\prime}}^{\infty}$. Then $A_{\theta^{\prime}}^{\infty} / K(J)$ is completely Morita equivalent to $S(G ; \sigma)$.

Note that there is a $*$-homomorphism $\psi_{*}$ from $A_{\theta}=C^{*}\left(\mathbb{Z}^{n} ; \sigma_{\theta}\right)$ onto $C^{*}(G ; \sigma)$ sending $u_{g}$ to $\lambda_{g} u_{\psi(g)}$ for all $g \in \mathbb{Z}^{n}$. From the universal property of $C^{*}(G ; \sigma)$ one sees that $\operatorname{ker}\left(\psi_{*}\right)$ is the closed ideal of $C^{*}\left(\mathbb{Z}^{n} ; \sigma_{\theta}\right)$ generated by $u_{g_{j}}-\lambda_{g_{j}} \lambda_{0}^{-1}, 1 \leq j \leq m$ for any basis $g_{1}, \cdots, g_{m}$ for $\operatorname{ker}(\psi)$. Denote $u_{g_{j}}-\lambda_{g_{j}} \lambda_{0}^{-1}$ by $v_{j}$. Observing that $v_{j}$ is in the center of $C^{*}\left(\mathbb{Z}^{n} ; \sigma_{\theta}\right)$, we see that $\operatorname{ker}\left(\psi_{*}\right)$ is the closure of $\sum_{j=1}^{m} v_{j} C^{*}\left(\mathbb{Z}^{n} ; \sigma_{\theta}\right)$ in $C^{*}\left(\mathbb{Z}^{n} ; \sigma_{\theta}\right)$. Denote by $J$ the intersection of $S\left(\mathbb{Z}^{n} ; \sigma_{\theta}\right)$ and $\operatorname{ker}\left(\psi_{*}\right)$. Then $J$ is a closed two-sided ideal of $S\left(\mathbb{Z}^{n} ; \sigma_{\theta}\right)$ and is the closure of $\sum_{j=1}^{m} v_{j} S\left(\mathbb{Z}^{n} ; \sigma_{\theta}\right)$ in $S\left(\mathbb{Z}^{n} ; \sigma_{\theta}\right)$. Using $\psi$ we may identify $\hat{G}$ with a closed subgroup of $\widehat{\mathbb{Z}^{n}}$, and hence identify $\operatorname{Lie}(\hat{G})$ with a Lie subalgebra of Lie $\left(\widehat{\mathbb{Z}^{n}}\right)$. Clearly, $\psi_{*}$ sends $A_{\theta}^{\infty}=S\left(\mathbb{Z}^{n} ; \sigma_{\theta}\right)$ into $S(G ; \sigma)$. It follows that $\operatorname{Lie}(\hat{G})$ preserves $J$. It is easily checked that elements of $S(G ; \sigma)$ are of the form $\sum_{g \in G} c_{g} u_{g}$ for the coefficient function $G \ni g \mapsto c_{g}$ belonging to the Schwarz space $\mathcal{S}(G)$ (cf. the proof of the corollary 
on page 468 of $[22])$. Thus $\psi_{*}$ sends $S\left(\mathbb{Z}^{n} ; \sigma_{\theta}\right)$ onto $S(G ; \sigma)$. Consequently, $A_{\theta}^{\infty} / J$ and $S(G ; \sigma)$ are isomorphic as pre- $C^{*}$-algebras, in a way compatible with the actions of $\operatorname{Lie}(\hat{G})$.

Now we need to find the corresponding ideal $K(J)$ of $A_{\theta^{\prime}}^{\infty}$. There is a $*$-isomorphism $\varphi$ from the center of $A_{\theta}^{\infty}$ onto that of $A_{\theta^{\prime}}^{\infty}$ determined by $\varphi(a) x=x a$ for all $a \in A_{\theta}^{\infty}$ and $x \in E$. Clearly, the corresponding closed $A_{\theta^{\prime}}^{\infty}-A_{\theta}^{\infty}$-submodule $Y$ of $E$ is the closure of $\sum_{j=1}^{m} E v_{j}$ in $E$, and $K(J)$ is the closed two-sided ideal of $A_{\theta^{\prime}}^{\infty}$ generated by $\varphi\left(v_{j}\right), 1 \leq j \leq m$. Recall that the center of $A_{\theta}^{\infty}=S\left(G ; \sigma_{\theta}\right)$ is the closed linear span of $u_{g}, g \in H_{\sigma_{\theta}}$, in $S\left(G ; \sigma_{\theta}\right)$ (see Subsection 2.2). Moreover, the eigenvectors of the restriction of the action of $\operatorname{Lie}\left(\widehat{\mathbb{Z}^{n}}\right)$ on $S\left(\mathbb{Z}^{n} ; \sigma_{\theta}\right)$ to the center are exactly the scalar multiples of the $u_{g}$ 's for $g \in H_{\sigma_{\theta}}$. Therefore, for any $g \in H_{\sigma_{\theta}}$, up to a scalar multiple, $\varphi\left(u_{g}\right)$ is equal to $u_{g^{\prime}}$ for some $g^{\prime} \in H_{\sigma_{\theta^{\prime}}}$. The map $\eta$ from $H_{\sigma_{\theta}}$ to $H_{\sigma_{\theta^{\prime}}}$ sending $g$ to $g^{\prime}$ is easily seen to be an isomorphism. Consequently, $K(J)$ is the closed two-sided ideal of $A_{\theta^{\prime}}^{\infty}$ generated by $u_{\varphi\left(g_{j}\right)}-\gamma_{j}, 1 \leq j \leq m$, where $\gamma_{j}$ is a certain element of $\mathbb{T}$. Note that $\operatorname{ker}(\psi) \subseteq H_{\sigma_{\theta}}$ and $\mathbb{Z}^{n-k} \oplus 0^{k}=H_{\sigma_{\theta^{\prime}}}$. Set $G^{\prime}=\mathbb{Z}^{n} / \eta(\operatorname{ker}(\psi))$. Then $G^{\prime}=G_{1}^{\prime} \oplus G_{2}^{\prime}$ for $G_{1}^{\prime}=H_{\sigma_{\theta^{\prime}}} / \eta(\operatorname{ker}(\psi))$ and $G_{2}^{\prime}=\mathbb{Z}^{k}$. Set $\sigma^{\prime}$ equal to the skew-symmetric bicharacter on $G^{\prime}$ such that $\sigma^{\prime}\left(G_{1}^{\prime}, G^{\prime}\right)=1$ and the restrictions of $\sigma^{\prime}$ and $\sigma_{\theta^{\prime}}$ to $G_{2}^{\prime}$ coincide. Then the pull-back of $\sigma^{\prime}$ under the quotient map $\psi^{\prime}$ from $\mathbb{Z}^{n}$ to $G^{\prime}$ is exactly $\sigma_{\theta^{\prime}}$, and the restriction of $\sigma^{\prime}$ to $\mathbb{Z}^{k}$ is nondegenerate. Note that for any basis $h_{1}, \cdots, h_{n-k}$ for $\mathbb{Z}^{n-k}$ and any $\mu_{1}, \cdots, \mu_{n-k} \in \mathbb{T}$ there is a $*$-homomorphism from $C^{*}\left(\mathbb{Z}^{n} ; \sigma_{\theta^{\prime}}\right)=A_{\theta^{\prime}}$ onto $C^{*}\left(G^{\prime} ; \sigma^{\prime}\right)$ sending $u_{h_{j}}$ to $\mu_{j} u_{\psi\left(h_{j}\right)}$ for all $1 \leq j \leq k$ and sending $u_{h}$ to $u_{\psi(h)}$ for all $h \in \mathbb{Z}^{k}$. Using the elementary divisor theorem [20, Theorem III.7.8], we can choose suitable $\mu_{1}, \cdots, \mu_{n-k}$ such that the kernel of the above $*$-homomorphism is exactly the closed twosided ideal of $C^{*}\left(\mathbb{Z}^{n} ; \sigma_{\theta^{\prime}}\right)$ generated by $u_{\varphi\left(g_{j}\right)}-\gamma_{j}, 1 \leq j \leq m$. Then $K(J)$ is the intersection of this ideal and $S\left(\mathbb{Z}^{n} ; \sigma_{\theta^{\prime}}\right)=A_{\theta^{\prime}}^{\infty}$. As in the last paragraph, we may identify $\operatorname{Lie}\left(\widehat{G^{\prime}}\right)$ with a Lie subalgebra of $\operatorname{Lie}\left(\mathbb{Z}^{n}\right)$, and $K(J)$ is invariant under the action of $\operatorname{Lie}\left(\widehat{G^{\prime}}\right)$. Furthermore, $A_{\theta^{\prime}}^{\infty} / K(J)$ and $S\left(G^{\prime} ; \sigma^{\prime}\right)$ are isomorphic as pre- $C^{*}$-algebras, in a way compatible with the actions of $\operatorname{Lie}\left(\widehat{G^{\prime}}\right)$. Observing that $\operatorname{Lie}(\hat{G})$ (resp. Lie $\left.\left(\widehat{G^{\prime}}\right)\right)$ consists of exactly those elements of $\operatorname{Lie}\left(\widehat{\mathbb{Z}^{n}}\right)$ (resp. Lie $\left(\widehat{\mathbb{Z}^{n}}\right)$ ) acting trivially on the center of $A_{\theta}^{\infty}$ (resp. $A_{\theta^{\prime}}^{\infty}$ ), we see that $\operatorname{Lie}(\hat{G})$ is sent onto $\operatorname{Lie}\left(\widehat{G^{\prime}}\right)$ under the Lie algebra isomorphism $\phi: \operatorname{Lie}\left(\widehat{\mathbb{Z}^{n}}\right) \rightarrow \operatorname{Lie}\left(\widehat{\mathbb{Z}^{n}}\right)$. Therefore, $S\left(G^{\prime} ; \sigma^{\prime}\right)$ and $S(G ; \sigma)$ are completely Morita equivalent. From $\operatorname{rank}(G)+\operatorname{rank}(\operatorname{ker}(\psi))=\operatorname{rank}\left(\mathbb{Z}^{n}\right)$ and 
$\operatorname{rank}\left(G^{\prime}\right)+\operatorname{rank}(\eta(\operatorname{ker}(\psi)))=\operatorname{rank}\left(\mathbb{Z}^{n}\right)$ we obtain $\operatorname{rank}(G)=\operatorname{rank}\left(G^{\prime}\right)$. This finishes the proof of Theorem 5.1.

Recall the group $H_{\sigma}$ and the skew-symmetric bicharacter $\sigma^{*}$ defined in Subsection 2.2 for a 2-cocycle $\sigma$ on a finitely generated abelian group $G$.

Remark 5.2. We indicate briefly another proof of Theorem 5.1. Note that $\sigma^{*}$ induces a skew-symmetric bicharacter $\tau^{*}$ on $G^{\prime \prime}:=G /\left(H_{\sigma}\right)_{\text {tor }}$. Then $\tau^{*}=\left(\sigma^{\prime \prime}\right)^{*}$ for some 2-cocycle $\sigma^{\prime \prime}$ on $G^{\prime \prime}$. Since $\widehat{G^{\prime \prime}}$ is a subgroup of $\hat{G}$, it also acts on $C^{*}(G ; \sigma)$. One checks easily that $C^{*}(G ; \sigma)$ and the direct sum of $\left|\left(H_{\sigma}\right)_{\text {tor }}\right|$ many copies of $C^{*}\left(G^{\prime \prime} ; \sigma^{\prime \prime}\right)$ are isomorphic in a way compatible with the actions of $\widehat{G^{\prime \prime}}$. Using the proof of [34, Proposition], by induction on $\left|G_{\text {tor }}^{\prime \prime}\right|$, one can show that there is a free (abelian) subgroup $G^{\prime}$ of $G^{\prime \prime}$ of the same rank as $G$ such that $C^{*}\left(G^{\prime \prime} ; \sigma^{\prime \prime}\right)$ is isomorphic to $M_{m}\left(C^{*}\left(G^{\prime} ; \sigma^{\prime}\right)\right)$ for some $m$ and $S\left(G^{\prime \prime} ; \sigma^{\prime \prime}\right)$ is completely Morita equivalent to $S\left(G^{\prime} ; \sigma^{\prime}\right)$, where $\sigma^{\prime}$ is the restriction of $\sigma^{\prime \prime}$ to $G^{\prime}$. Consequently, $C^{*}(G ; \sigma)$ is isomorphic to the direct sum of $\left|\left(H_{\sigma}\right)_{\text {tor }}\right|$ many copies of $M_{m}\left(C^{*}\left(G^{\prime} ; \sigma^{\prime}\right)\right)$ and $S(G ; \sigma)$ is completely Morita equivalent to the direct sum of $\left|\left(H_{\sigma}\right)_{\text {tor }}\right|$ many copies of $S\left(G^{\prime} ; \sigma^{\prime}\right)$. Then Theorem 5.1 follows from Theorem 3.1. However, the earlier detailed proof does not use induction and is more likely to be generalizable to the case of arbitrary (countable) discrete abelian groups.

Lemma 5.3. In Theorem 5.1 one has $\left|\left(H_{\sigma}\right)_{\text {tor }}\right|=\left|\left(G_{1}^{\prime}\right)_{\text {tor }}\right|$ and $\operatorname{rank}\left(H_{\sigma}\right)=$ $\operatorname{rank}\left(G_{1}^{\prime}\right)$.

Proof. Recall that the center of $C^{*}(G ; \sigma)$ is isomorphic to $C\left(\widehat{H_{\sigma}}\right)$. Note that $\left|\left(H_{\sigma}\right)_{\text {tor }}\right|$ and $\operatorname{rank}\left(H_{\sigma}\right)$ are the number of connected components and the dimension of $\widehat{H_{\sigma}}$ respectively. Since Morita equivalence preserves centers [1, Proposition 21.10], we obtain $\left|\left(H_{\sigma}\right)_{\text {tor }}\right|=\left|\left(H_{\sigma^{\prime}}\right)_{\text {tor }}\right|=$ $\left|\left(G_{1}^{\prime}\right)_{\text {tor }}\right|$ and $\operatorname{rank}\left(H_{\sigma}\right)=\operatorname{rank}\left(H_{\sigma^{\prime}}\right)=\operatorname{rank}\left(G_{1}^{\prime}\right)$.

From the Pimsner-Voiculescu exact sequence [26] one knows that the $K_{0}$-group (resp. $K_{1}$-group) of an $n$-dimensional noncommutative torus is a free abelian group of rank $2^{n-1}$ or 1 (resp. $2^{n-1}$ or 0 ) depending as $n>0$ or $n=0$. Since strong Morita equivalence between $C^{*}$-algebras preserves $K$-groups [7, Theorem 1.2], [15], we get

Corollary 5.4. Let $\sigma$ be a 2-cocycle on a finitely generated abelian group $G$. Then the $K_{0}$-group (resp. $K_{1}$-group) of $C^{*}(G ; \sigma)$ is a free abelian group with rank $\left|\left(H_{\sigma}\right)_{\mathrm{tor}}\right| \cdot 2^{\operatorname{rank}(G)-1}$ or $\left|\left(H_{\sigma}\right)_{\mathrm{tor}}\right|$ (resp. $\left|\left(H_{\sigma}\right)_{\mathrm{tor}}\right| \cdot$ $2^{\operatorname{rank}(G)-1}$ or 0$)$ depending as $\operatorname{rank}(G)>0$ or $\operatorname{rank}(G)=0$. 
For any unital $C^{*}$-algebra $A$, denote by $T(A)_{K_{0}}$ the set of all homomorphisms from $K_{0}(A)$ to $\mathbb{R}$ induced by tracial states of $A$. Then $T(A)_{K_{0}}$ equipped with the topology of pointwise convergence is a compact convex set in a Hausdorff locally convex topological vector space.

Lemma 5.5. Let $\sigma$ be a 2-cocycle on a finitely generated abelian group $G$. Then $T\left(C^{*}(G ; \sigma)\right)_{K_{0}}$ is a simplex of dimension $\left|\left(H_{\sigma}\right)_{\text {tor }}\right|-1$. The images of $K_{0}\left(C^{*}(G ; \sigma)\right)$ are the same under all the vertices of $T\left(C^{*}(G ; \sigma)\right)_{K_{0}}$.

Proof. Recall the skew-symmetric bicharacter $\sigma^{*}$ on $G$ defined in Subsection 2.2. It induces a skew-symmetric bicharacter $\tau^{*}$ on $G / H_{\sigma}$. Then $\tau^{*}=\left(\sigma^{\prime}\right)^{*}$ for some 2-cocycle $\sigma^{\prime}$ on $G / H_{\sigma}$. Note that $\sigma^{\prime}$ is nondegenerate. Thus $C^{*}\left(G / H_{\sigma} ; \sigma^{\prime}\right)$ has a unique tracial state $\varphi$ [39, Lemma 3.2]. For any closed ideal $I_{t}$ of $C^{*}(G ; \sigma)$ generated by a maximal ideal $t$ of the center of $C^{*}(G ; \sigma)$, one has $C^{*}(G ; \sigma) / I_{t} \cong C^{*}\left(G / H_{\sigma} ; \sigma^{\prime}\right)$.

Any extremal point of $T\left(C^{*}(G ; \sigma)\right)_{K_{0}}$ is induced by an extremal tracial state of $C^{*}(G ; \sigma)$. An argument similar to that in the proof of [13, Lemma 2.2] shows that every extremal tracial state of $C^{*}(G ; \sigma)$ factors through $C^{*}(G ; \sigma) / I_{t}$ for some maximal ideal $t$ of the center (and hence must be the pull-back $\varphi_{t}$ of the unique tracial state of $\left.C^{*}(G ; \sigma) / I_{t}\right)$, and also that the map from $\widehat{H_{\sigma}}$ to $T\left(C^{*}(G ; \sigma)\right)_{K_{0}}$ sending $t$ to the homomorphism from $K_{0}\left(C^{*}(G ; \sigma)\right)$ to $\mathbb{R}$ induced by $\varphi_{t}$ is locally constant. Denote by $X$ the image of this map. Then $X$ has cardinality at most the number of components of $\widehat{H_{\sigma}}$, i.e., $\left|\left(H_{\sigma}\right)_{\text {tor }}\right|$. Also, $X$ contains all the extremal points of $T\left(C^{*}(G ; \sigma)\right)_{K_{0}}$ and hence the closed convex hull of $X$ is $T\left(C^{*}(G ; \sigma)\right)_{K_{0}}$ by the Krein-Milman theorem [11, Theorem V.7.4]. Evaluating elements of $X$ at minimal projections in the center of $C^{*}(G ; \sigma)$ we see that the closed convex hull of $X$ is a simplex of dimension $\left|H_{\sigma}\right|-1$ with vertex set $X$. This proves the first assertion of Lemma 5.5.

Note that if ${ }_{A} E_{B}$ is a strong Morita equivalence bimodule for two unital $C^{*}$-algebras $A$ and $B$, and $K(J), J, Y$ are closed two-sided ideals and a submodule as in Subsection 2.1, then the diagram

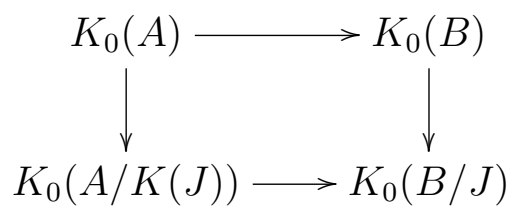

commutes, where the horizonal isomorphisms are induced by ${ }_{A} E_{B}$ and ${ }_{A / K(J)} E / Y_{B / J}$ respectively and the vertical homomorphisms are induced by the $C^{*}$-algebra quotient maps. Using Theorem 5.1 one sees 
that the homomorphism from $K_{0}\left(C^{*}(G ; \sigma)\right)$ to $K_{0}\left(C^{*}(G ; \sigma) / I_{t}\right)$ induced by the $C^{*}$-algebra quotient map is surjective for every maximal ideal $t$ of the center of $C^{*}(G ; \sigma)$. Thus the image of $K_{0}\left(C^{*}(G ; \sigma)\right)$ under any element of $X$ is the image of $K_{0}\left(C^{*}\left(G / H_{\sigma} ; \sigma^{\prime}\right)\right)$ under the element of $T\left(C^{*}\left(G / H_{\sigma} ; \sigma^{\prime}\right)\right)_{K_{0}}$ induced by $\varphi$. This finishes the proof of Lemma 5.5.

Let $\omega_{\sigma}$ be any vertex of $T\left(C^{*}(G ; \sigma)\right)_{K_{0}}$ in Lemma 5.5. The following result is a generalization of Proposition 4.1.

Proposition 5.6. Let $\sigma_{j}$ be a 2-cocycle on a finitely generated abelian group $G_{j}$ for $j=1,2$. Then $C^{*}\left(G_{1} ; \sigma_{1}\right)$ and $C^{*}\left(G_{2} ; \sigma_{2}\right)$ have isomorphic ordered $K_{0}$-groups if and only if $\omega_{\sigma_{2}}\left(K_{0}\left(C^{*}\left(G_{2} ; \sigma_{2}\right)\right)\right)=\mu \omega_{\sigma_{1}}\left(K_{0}\left(C^{*}\left(G_{1} ; \sigma_{1}\right)\right)\right)$ for some real number $\mu>0,\left|\left(H_{\sigma_{1}}\right)_{\text {tor }}\right|=\left|\left(H_{\sigma_{2}}\right)_{\text {tor }}\right|$, and $\operatorname{rank}\left(G_{1}\right)=$ $\operatorname{rank}\left(G_{2}\right)$ or $\operatorname{rank}\left(G_{1}\right)+\operatorname{rank}\left(G_{2}\right)=1$.

Proof. Let us prove first the "only if" part. An argument similar to that in the proof of the "only if" part of Proposition 4.1 shows that $\omega_{\sigma_{2}}\left(K_{0}\left(C^{*}\left(G_{2} ; \sigma_{2}\right)\right)\right)=\mu \omega_{\sigma_{1}}\left(K_{0}\left(C^{*}\left(G_{1} ; \sigma_{1}\right)\right)\right)$ for some real number $\mu>0$. By Lemma 5.5 the set of positive homomorphisms from $\left(K_{0}\left(C^{*}\left(G_{j} ; \sigma_{j}\right)\right)_{+}, K_{0}\left(C^{*}\left(G_{j} ; \sigma_{j}\right)\right)\right)$ to $\left(\mathbb{R}_{+}, \mathbb{R}\right)$ is a cone of dimension $\left|\left(H_{\sigma_{j}}\right)_{\text {tor }}\right|$. Thus, $\left|\left(H_{\sigma_{1}}\right)_{\text {tor }}\right|=\left|\left(H_{\sigma_{2}}\right)_{\text {tor }}\right|$. Comparing the ranks of the $K_{0}$-groups, by Corollary 5.4 we obtain $\operatorname{rank}\left(G_{1}\right)=\operatorname{rank}\left(G_{2}\right)$ or $\operatorname{rank}\left(G_{1}\right)+\operatorname{rank}\left(G_{2}\right)=1$.

For the "if" part, by Theorem 5.1 and the "only if" part we may assume that $G_{j}$ and $\sigma_{j}$ have the same properties as $G^{\prime}$ and $\sigma^{\prime}$ of Theorem 5.1. Then an argument similar to that in the proof of the "if" part of Proposition 4.1 completes the proof.

An argument similar to that in the proof of Lemma 4.3 establishes the following generalization of Lemma 4.3:

Lemma 5.7. Let $\sigma_{j}$ be a skew-symmetric bicharacter on a finitely generated abelian group $G_{j}$ for $j=1,2$ such that $G_{j}=G_{j}^{\prime \prime} \oplus G_{j}^{\prime}$, $\sigma_{j}\left(G_{j}^{\prime \prime}, G_{j}\right)=1$, and $G_{j}^{\prime}$ is torsion-free. Denote by $\sigma_{j}^{\prime}$ the restriction of $\sigma_{j}$ to $G_{j}^{\prime}$. Suppose that $\operatorname{rank}\left(G_{1}^{\prime}\right)=\operatorname{rank}\left(G_{2}^{\prime}\right)$. If the ordered $K_{0}$ groups of $C^{*}\left(G_{1} ; \sigma_{1}\right)$ and $C^{*}\left(G_{2} ; \sigma_{2}\right)$ are isomorphic, then so also are those of $C^{*}\left(G_{1}^{\prime} ; \sigma_{1}^{\prime}\right)$ and $C^{*}\left(G_{2}^{\prime} ; \sigma_{2}^{\prime}\right)$.

Now the proof of Theorem 1.1 in the case of noncommutative tori in Section 4 extends verbatim to the general case of the twisted group $C^{*}$-algebras of arbitrary finitely generated abelian groups.

Remark 5.8. For a 2-cocycle $\sigma$ on a finitely generated abelian group $G$, one can check easily that the maximal ideals of $C^{*}(G ; \sigma)$ are exactly 
those $I_{t}$ 's in the proof of Lemma 5.5, and hence that the simple quotient algebras of $C^{*}(G ; \sigma)$ are all isomorphic to $C^{*}\left(G / H_{\sigma} ; \sigma^{\prime}\right)$ therein. The proof of Theorem 5.1 actually shows that two twisted group $C^{*}$-algebras of finitely generated abelian groups are strongly Morita equivalent if and only if they have isomorphic centers and their simple quotient algebras have isomorphic ordered $K_{0}$-groups.

\section{REFERENCES}

[1] F. W. Anderson and K. R. Fuller. Rings and Categories of Modules. Second edition. Graduate Texts in Mathematics, 13. Springer-Verlag, New York, 1992.

[2] B. Blackadar. K-theory for Operator Algebras. Second edition. Mathematical Sciences Research Institute Publications, 5. Cambridge University Press, Cambridge, 1998.

[3] B. Blackadar and M. Rørdam. Extending states on preordered semigroups and the existence of quasitraces on $C^{*}$-algebras. J. Algebra 152 (1992), no. 1, 240247.

[4] W. Beer. On Morita equivalence of nuclear $C^{*}$-algebras. J. Pure Appl. Algebra 26 (1982), no. 3, 249-267.

[5] O. Bratteli, G. A. Elliott, and P. E. T. Jorgensen. Decomposition of unbounded derivations into invariant and approximately inner parts. J. Reine Angew. Math. 346 (1984), 166-193.

[6] B. Brenken, J. Cuntz, G. A. Elliott, and R. Nest. On the classification of noncommutative tori. III. In: Operator Algebras and Mathematical Physics (Iowa City, Iowa, 1985), 503-526, Contemp. Math., 62, Amer. Math. Soc., Providence, RI, 1987.

[7] L. G. Brown, P. Green, and M. A. Rieffel. Stable isomorphism and strong Morita equivalence of $C^{*}$-algebras. Pacific J. Math. 71 (1977), no. 2, 349-363.

[8] A. Connes. $C^{*}$-algèbres et géométrie différentielle. C. R. Acad. Sci. Paris Sér. A-B 290 (1980), no. 13, A599-A604. hep-th/0101093.

[9] A. Connes. Noncommutative Geometry. Academic Press, Inc., San Diego, CA, 1994.

[10] A. Connes, M. R. Douglas, and A. Schwarz. Noncommutative geometry and matrix theory: compactification on tori. J. High Energy Phys. 1998, no. 2, Paper 3, 35 pp. (electronic). hep-th/9711162.

[11] J. B. Conway. A Course in Functional Analysis. Second edition. Graduate Texts in Mathematics, 96. Springer-Verlag, New York, 1990.

[12] S. Doplicher, R. Longo, J. E. Roberts, and L. Zsidó. A remark on quantum group actions and nuclearity. Dedicated to Professor Huzihiro Araki on the occasion of his 70th birthday. Rev. Math. Phys. 14 (2002), no. 7-8, 787-796. math.OA/0204029.

[13] G. A. Elliott. On the $K$-theory of the $C^{*}$-algebra generated by a projective representation of a torsion-free discrete abelian group. In: Operator Algebras and Group Representations, Vol. I (Neptun, 1980), 157-184, Monogr. Stud. Math., 17, Pitman, Boston, MA, 1984.

[14] G. A. Elliott and H. Li. Morita equivalence of smooth noncommutative tori. Acta Math. 199 (2007), 1-27. math.OA/0311502. 
[15] R. Exel. A Fredholm operator approach to Morita equivalence. $K$-Theory 7 (1993), no. 3, 285-308.

[16] U. Haagerup and S. Thorbjørnsen. Random matrices and $K$-theory for exact $C^{*}$-algebras. Doc. Math. 4 (1999), 341-450 (electronic).

[17] E. Kissin and V. S. Shul'man. Dense $Q$-subalgebras of Banach and $C^{*}$-algebras and unbounded derivations of Banach and $C^{*}$-algebras. Proc. Edinburgh Math. Soc. (2) 36 (1993), no. 2, 261-276.

[18] A. Kleppner. Multipliers on abelian groups. Math. Ann. 158 (1965), 11-34.

[19] A. Konechny and A. Schwarz. Introduction to M(atrix) theory and noncommutative geometry. Phys. Rep. 360 (2002), no. 5-6, 353-465.

[20] S. Lang. Algebra. Revised third edition. Graduate Texts in Mathematics, 211. Springer-Verlag, New York, 2002.

[21] H. Li. Strong Morita equivalence of higher-dimensional noncommutative tori. J. Reine Angew. Math. 576 (2004), 167-180. math.OA/0303123.

[22] P. Milnes and S. Walters. $C^{*}$-algebras characterized by ergodic actions of the n-torus. Bull. London Math. Soc. 32 (2000), no. 4, 465-470.

[23] D. Olesen, G. K. Pedersen, and M. Takesaki. Ergodic actions of compact abelian groups. J. Operator Theory 3 (1980), no. 2, 237-269.

[24] N. C. Phillips. Continuous-trace $C^{*}$-algebras not isomorphic to their opposite algebras. Internat. J. Math. 12 (2001), no. 3, 263-275.

[25] N. C. Phillips. Every simple higher dimensional noncommutative torus is an AT algebra. math.OA/0609783.

[26] M. Pimsner and D. Voiculescu. Exact sequences for $K$-groups and Ext-groups of certain cross-product $C^{*}$-algebras. J. Operator Theory 4 (1980), no. 1, 93118.

[27] M. A. Rieffel. Induced representations of $C^{*}$-algebras. Advances in Math. 13 (1974), $176-257$.

[28] M. A. Rieffel. Unitary representations of group extensions; an algebraic approach to the theory of Mackey and Blattner. Studies in Analysis, pp. 43-82, Adv. in Math. Suppl. Stud., 4, Academic Press, New York-London, 1979.

[29] M. A. Rieffel. $C^{*}$-algebras associated with irrational rotations. Pacific J. Math. 93 (1981), no. 2, 415-429.

[30] M. A. Rieffel. Morita equivalence for operator algebras. In: Operator Algebras and Applications, Part I (Kingston, Ont., 1980), pp. 285-298, Proc. Sympos. Pure Math., 38, Amer. Math. Soc., Providence, R.I., 1982.

[31] M. A. Rieffel. Projective modules over higher dimensional noncommutative tori. Canad. J. Math. 40 (1988), no. 2, 257-338.

[32] M. A. Rieffel. Non-commutative tori - a case study of non-commutative differentiable manifolds. In: Geometric and Topological Invariants of Elliptic Operators (Brunswick, ME, 1988), 191-211, Contemp. Math., 105, Amer. Math. Soc., Providence, RI, 1990.

[33] M. A. Rieffel and A. Schwarz. Morita equivalence of multidimensional noncommutative tori. Internat. J. Math. 10 (1999), no. 2, 289-299. math.QA/9803057.

[34] J. Rosenberg. Appendix to: "Crossed products of UHF algebras by product type actions" [Duke Math. J. 46 (1979), no. 1, 1-23] by O. Bratteli. Duke Math. J. 46 (1979), no. 1, 25-26.

[35] A. Schwarz. Morita equivalence and duality. Nuclear Phys. B 534 (1998), no. 3, 720-738. hep-th/9805034. 
[36] A. Schwarz. Noncommutative supergeometry and duality. Lett. Math. Phys. 50 (1999), no. 4, 309-321. hep-th/9912212.

[37] A. Schwarz. Noncommutative supergeometry, duality and deformations. $\mathrm{Nu}$ clear Phys. B 650 (2003), no. 3, 475-496. hep-th/0210271.

[38] N. Seiberg and E. Witten. String theory and noncommutative geometry. $J$. High Energy Phys. 1999, no. 9, Paper 32, 93 pp. (electronic). hep-th/9908142.

[39] J. Slawny. On factor representations and the $C^{*}$-algebra of canonical commutation relations. Comm. Math. Phys. 24 (1972), 151-170.

[40] X. Tang and A. Weinstein. Quantization and Morita equivalence for constant Dirac structures on tori. Ann. Inst. Fourier (Grenoble) 54 (2004), no. 5, 1-16. math.QA/0305413.

[41] F. Trèves. Topological Vector Spaces, Distributions and Kernels. Academic Press, New York-London 1967.

[42] G. Zeller-Meier. Produits croisés d'une $C^{*}$-algèbre par un groupe d'automorphismes. (French) J. Math. Pures Appl. (9) 47 (1968), 101-239.

Department of Mathematics, University of Toronto, Toronto, OnTARIO, CANADA M5S 2E4

E-mail address: elliott@math.toronto.edu

Department of Mathematics, SUNy at Buffalo, Buffalo, Ny 14260, USA

E-mail address: hfli@math.buffalo.edu 